\title{
Application of heterogeneous multiple camera system with panoramic capabilities in a Harbor Environment
}

\author{
Piet B.W. Schwering*, Henk A. Lensen, Sebastiaan P. van den Broek, Richard J.M. den Hollander, \\ Wannes van der Mark, Henri Bouma, Rob A.W. Kemp \\ TNO Defence, Security and Safety, P.O. Box 96864, 2509 JG The Hague, The Netherlands
}

\begin{abstract}
In a harbor environment threats like explosives-packed rubber boats, mine-carrying swimmers and divers must be detected in an early stage. This paper describes the integration and use of a heterogeneous multiple camera system with panoramic observation capabilities for detecting these small vessels in the Den Helder New Harbor in the Netherlands. Results of a series of experiments with different targets are presented. An outlook to a future sensor package containing panoramic vision is discussed. We also investigated several aspects of the use of electro-optical systems. As for classification, this paper concentrates on discriminating classes of small vessels with different electro-optical systems (visual and infrared) as part of the larger process involving an operator. It addresses both selection of features (based on shape and texture) and ways of using these in a system to assess threats. Results are presented on data recorded in coastal and harbor environments for several small targets.
\end{abstract}

Keywords: Panoramic vision, IRST segment, detection, image enhancement, image fusion, image stitching, classification, target recognition, small targets, boats, jet-ski, coastal environment.

\section{INTRODUCTION}

\subsection{Background}

History has proven that commercial and military vessels are vulnerable to asymmetric and volatile threats posed by terrorists and pirates. This threat is not only present at sea, as shown by the numerous attacks on commercial vessels in recent years [1], but also in harbors as was demonstrated by the attack on the USS Cole in 2000. The USS Cole was bombed in the harbor of Aden (Yemen) by a small craft approaching the ship from the port side killing 17 crewmen and damaging the ship's hull (Fig. 1)[2,3].

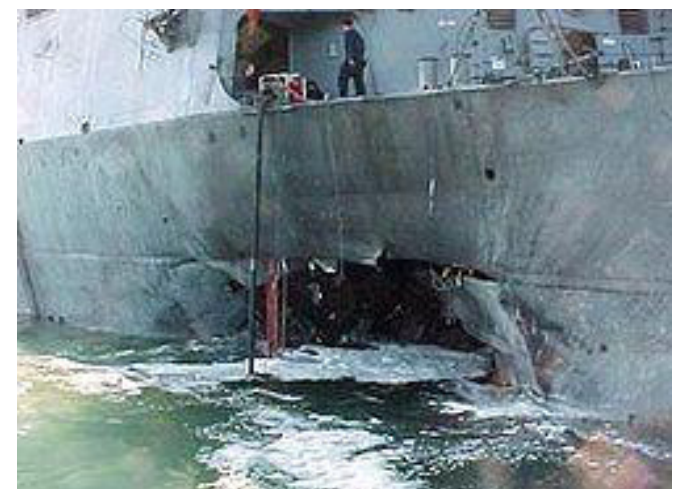

Fig. 1 Damaged hull of USS Cole [3]

Observation of these asymmetric threats at an early stage is indispensable to give the vessels sufficient time to react with appropriate measures. New techniques and approaches are required to support the crew to detect, classify and identify these threats, clock round against complex backgrounds and during adverse weather conditions.

*piet.schwering@tno.nl; phone 3170374 0470; fax 3170374 0654; www.tno.nl

Electro-Optical and Infrared Systems: Technology and Applications VI, edited by David A. Huckridge, Reinhard R. Ebert, Proc. of SPIE Vol. 7481, 74810C · C 2009 SPIE · CCC code: 0277-786X/09/\$18 - doi: 10.1117/12.833469 
Also, tasks of naval forces are more frequently taking place in coastal environments. Such environments introduce specific challenges in building an operational picture for situational awareness. Finding ships that are of interest for an operational task (such as intercepting smugglers or the ships that could pose a threat) is a challenging task. Performing those tasks is often done by a human operator, based on radar information and using electro-optical sensors for final identification. The operator may be helped by a system that adds information from these sensors automatically, assisting him in selecting targets for further inspection, and helping in classifying ships as targets of interest.

Aided target recognition is a process providing object information to an operator in order to enable him to perform his tasks better, which means:

1. Earlier: recognize objects of interest at an earlier time by providing information at a larger distance.

2. Better: provide more detailed information or make classification more specific, which allows people with less expertise or specific training to perform the tasks.

3. More: a larger area or more sensors can be used by the same operator, by presenting relevant information, which prevents operators from being overloaded with data and allows them to do their task for longer periods.

In a coastal scenario, an operator will be helped with partial information, as long as not too much is presented, and confidence in the information is high enough. This also means classification does not need to be perfect, as long as the number of false alarms is acceptable. For example, simple features, together with track information, may help to assess the status of many ships more quickly.

\subsection{Objectives}

A trial was defined to collect data in order to demonstrate the feasibility of new techniques and approaches to detect, track, recognize and identify small surface targets in and near a harbor environment.

For the purpose of the trial a sensor network had been set up consisting of a heterogeneous set of cameras with panoramic observation capabilities, radar and an automated identification system (AIS). The sensor network was used to collect data of small surface targets in and near the New Harbor of Den Helder, in the Netherlands, in October 2008.

The collected data will be used in several studies involving the target detection, clutter reduction, classification, fusion and cueing based on infrared images and radar measurements. The first approach uses the wide field-of-view infrared images and radar to produce plots (detections, contacts) that will be sent to their individual trackers (MT3, MT2) to build target tracks. The second approach uses the plots to send to another tracker (M6T, [4]) that first builds infrared and radar tracks and secondly fuses them. The final approach uses both infrared and radar plots first to fuse within the M6T tracker (i.e. plot fusion) and secondly to build the fused tracks (Fig. 2).

After building the infrared, radar or fused tracks, they can be used for further action, e.g. to cue narrow field-of-view cameras on the plots of interest (Fig. 2). Images of different narrow field-of-view cameras can be fused (image fusion), and merged into the wide field-of-view images (image stitching) to enhance target recognition and identification by the human operator. Moreover, automated systems will be able to determine more and more accurate object attributes, which on the one hand will contribute to automated management of cameras and radar, and on the other hand will contribute in the compilation of an improved common operational picture. The latter forms the basis for decision making processes and subsequent actions [5].

Studies involving target detection, tracking and classification based on infrared images and radar are carried out among others within TNO [6, 7]. Studies involving plot and track fusion are carried out among others within the current project [see acknowledgements]. The results of these studies, however, will be presented in future papers. In SPIE paper [8] results of new classification algorithms are discussed which make use of the collected data described in this paper. 


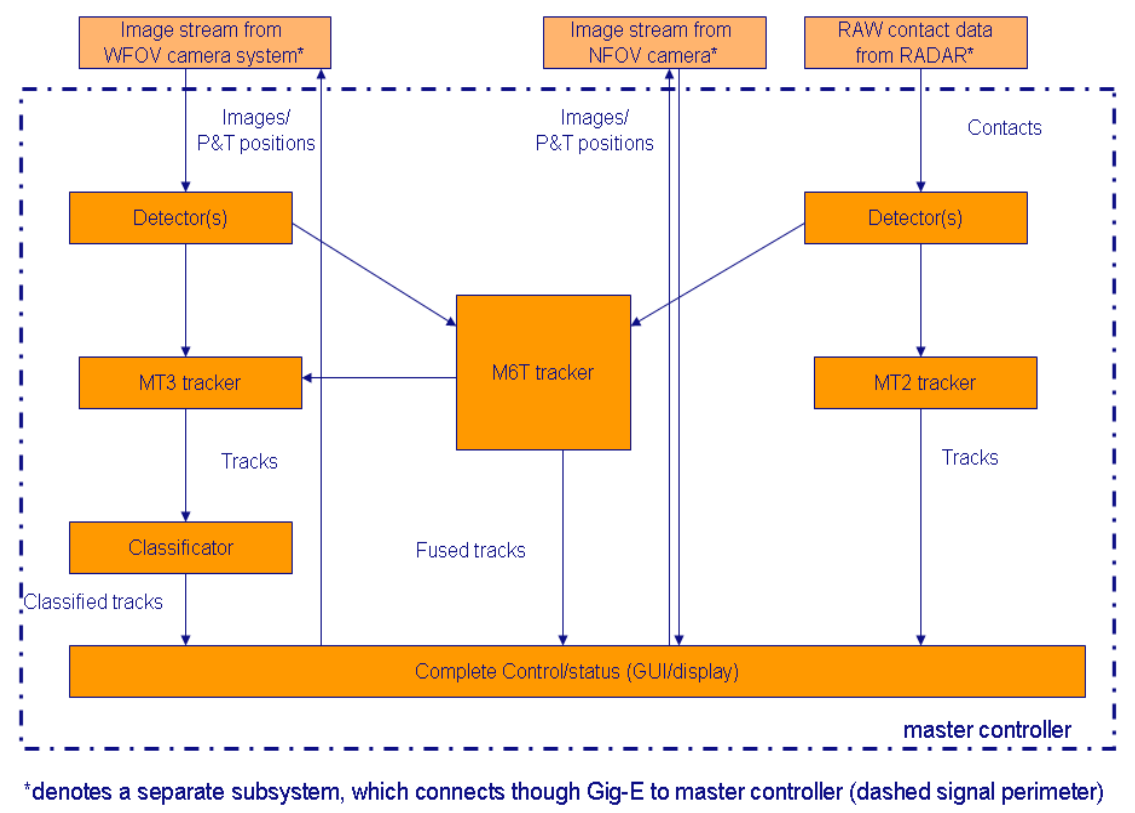

Fig. 2 Cueing, contact and track fusion scheme: Cueing of narrow field-of-view camera based on fused radar and wide fieldof-view camera plots (or tracks).

\subsection{Results}

This paper describes the trial setup including the attack scenarios of the targets, the sensors used, the data collection network and the sensor data acquired. In addition some examples are given of new approaches to present target image data in order to enhance situation awareness. Section 2 describes the classification processing chain that was assessed with the trials data. In Section 3 the trial setup, attack scenarios, sensors and data collection network are described. An overview of the classification processing is given in Section 4. Section 5 gives the processing results. Conclusions are drawn in Section 6.

\section{PROCESSING METHOD}

In this section different types of processing methods (based on different features) are described, at different stages of implementation. In Subsection 2.1, the processing chain is described, continuing previous work towards a real-time implementation. For generating the detections we made use of the algorithms developed by Bouma et al. [9]. Classification is performed on the regions of interest on the detected objects. A complete description of the method is given in van den Broek et al. [8].

\subsection{Processing chain}

Global descriptors are computed on the whole region of interest that is defined by a detection. These descriptors include normalized central moments, which are scale invariant $[10,11]$. These descriptors can be used to distinguish between clutter and surface targets, and the descriptors can be used to discriminate different types of targets. Classification is based on a binary decision tree and a mixture of Gaussians classifiers from PRTools [12].

A framework was generated that allows us to easily perform the following steps, as shown in Fig. 3. 


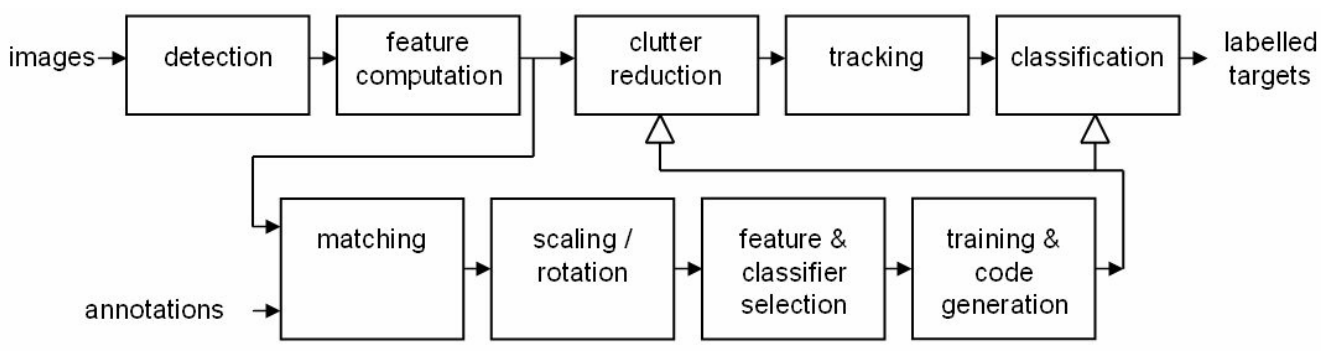

Fig. 3. Schematic overview of the processing chain. The top line is the near-realtime implementation. The bottom shows the offline processing performed to obtain the trained classifier.

The first step is real-time automatic detection of targets in the camera sequence. A detection algorithm provides us with bounding boxes in the image encapsulating surface targets, using robust estimation of the background intensity [9]. Ships are detected at various orientations, sizes and distances. After detection, the 'global' descriptors are computed in realtime for each detection.

Ground-truth labeling is performed manually (but aided) in the camera sequence. Assignment of class labels is needed to allow supervised learning. Boats are annotated in key frames and the labels are interpolated for intermediate frames. The labels are matched with the automatic detections to generate labeled detections.

Appropriate scaling and rotation of features is performed, e.g., by principal component analysis. For example, the performance of the nearest-neighbor classifier depends on the scaling between the different features, and the binarydecision-tree classifier depends on the orientation of the features.

\section{TRIAL SETUP}

A trial was defined to collect data in order to demonstrate the feasibility of new processing techniques and approaches to detect, track, classify and identify small surface targets in and near a harbor environment. For the purpose of the trial a littoral (offshore) and a harbor scenario were defined. Both scenarios were adapted from a generic scenario in which a docked frigate was attacked by small surface targets. The targets approached the frigate from the sea side or from inside the harbor.

\subsection{General attack scenario}

A target appears from behind an island and moves through a group of fishing vessels towards the frigate at slow speed. As soon as the target has left the group of fishing vessels, it accelerates and maintains a straight trajectory towards the frigate. The frigate detects and engages the target with gunfire as soon as it is within range of the surface gun. The target reacts with a weaving trajectory but maintains its general course towards the frigate. At first it carries out a weaving trajectory with a period of $20 \mathrm{~s}$ and as it comes closer the period increases $(40 \mathrm{~s})$. The speed of the target during the trajectories is constant.

\subsection{Trial scenarios}

In these scenarios the targets were sailing between the jetty of the isle of Texel and the harbor of Den Helder (littoral scenario) or within the confines of the harbor of Den Helder (harbor scenario). Target data like signature, position and velocity were collected using a heterogeneous set of cameras, radar and global positioning system (GPS). Where applicable AIS was used to gather information on other sailing objects. The cameras, radar and AIS were all located at the eastern pier of the harbor of Den Helder, near the collimation tower (Fig. 4). The GPS data loggers were fixed to the targets. 


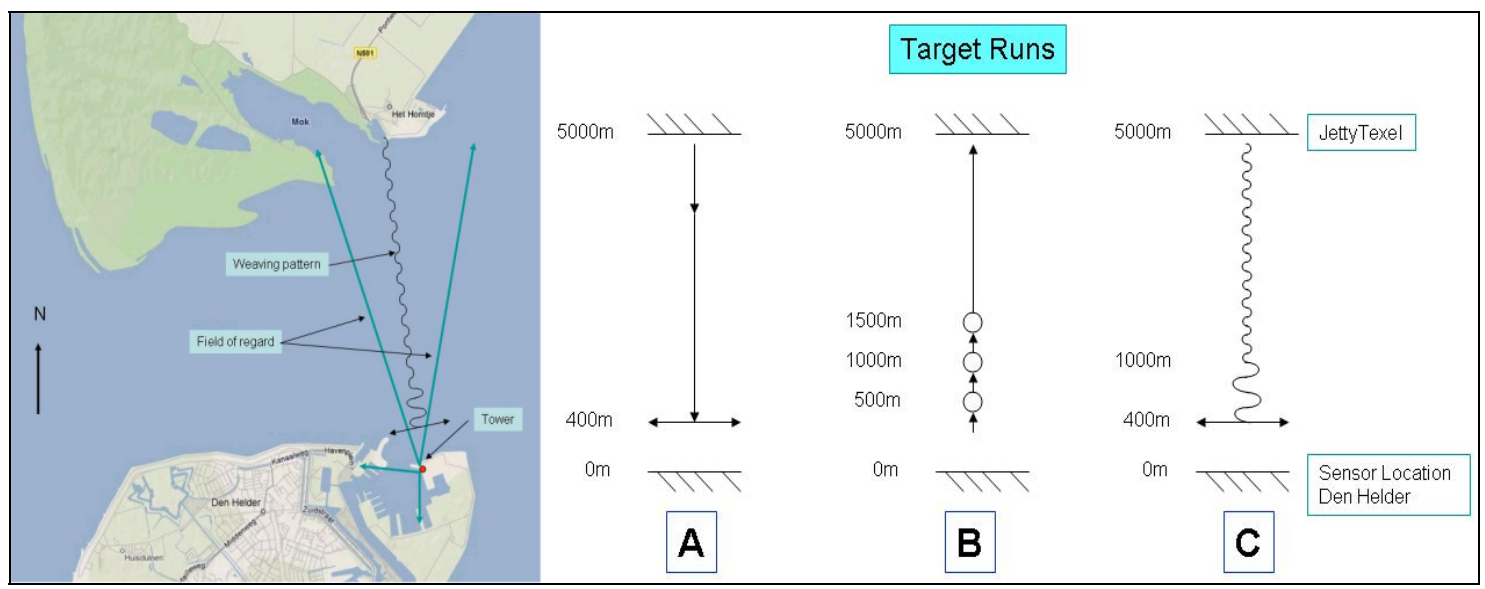

Fig. 4 Trial map of Den Helder and Texel (North). Measuring location is near the tower (left image, red dot). Right image shows for each target run three tracks: inbound straight line (A); outbound (B); inbound weaving (C).

\section{$\underline{\text { Littoral scenario }}$}

Each target run consisted out of three tracks: an inbound straight line (Fig. 4, A), an outbound (Fig. 4, B) and an inbound weaving pattern track (Fig. 4, C). For track (A) the targets started at the jetty of Texel (approximately $5 \mathrm{~km}$ North of the measurement location). The targets came inbound at high speed in a straight line towards the sensors to about $400 \mathrm{~m}$ before the pier, and then turned to the East to make a final beam-on passage from East to West. For track (B) the targets went outbound towards the Texel jetty starting in front of the sensors. During this track the targets had to make circles: at $500 \mathrm{~m}, 1000 \mathrm{~m}$ and $1500 \mathrm{~m}$. For track (C) the targets came inbound making a weaving pattern to abound $400 \mathrm{~m}$ before the measurement location, then turned to the East to make a final beam-on passage from East to West (second time). During this scenario the direction of the sensors is approximately North.

\section{Harbor scenario}

In this scenario no predefined tracks were used. One or two targets appeared from behind obstacles (other vessels) or near the quay and moved slowly towards the measurement location. At an unspecified moment the targets increased their speed towards the sensors simulating an attack.

\subsection{Targets}

Several small surface targets were arranged to sail the different attack scenarios. The targets arranged included two twin kayaks, a Landing Craft Rubber Motorized (LCRM), two rigid hull inflatable boats (RHIBs), two small patrol vessels and a jet-ski (Fig. 5, Fig. 6, Fig. 7). Furthermore several targets of opportunity passed the sensors' field-of-regard, among others the ferry (TESO) between Den Helder and Texel (Fig. 7).

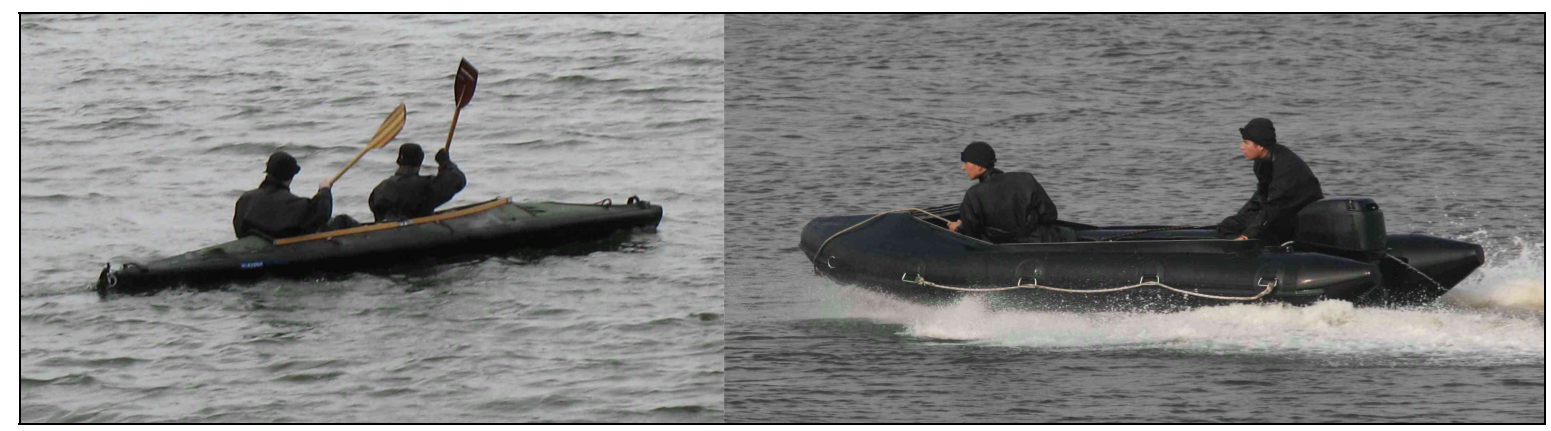

Fig. $5 \quad$ Kayak (left) and LCRM (right) 


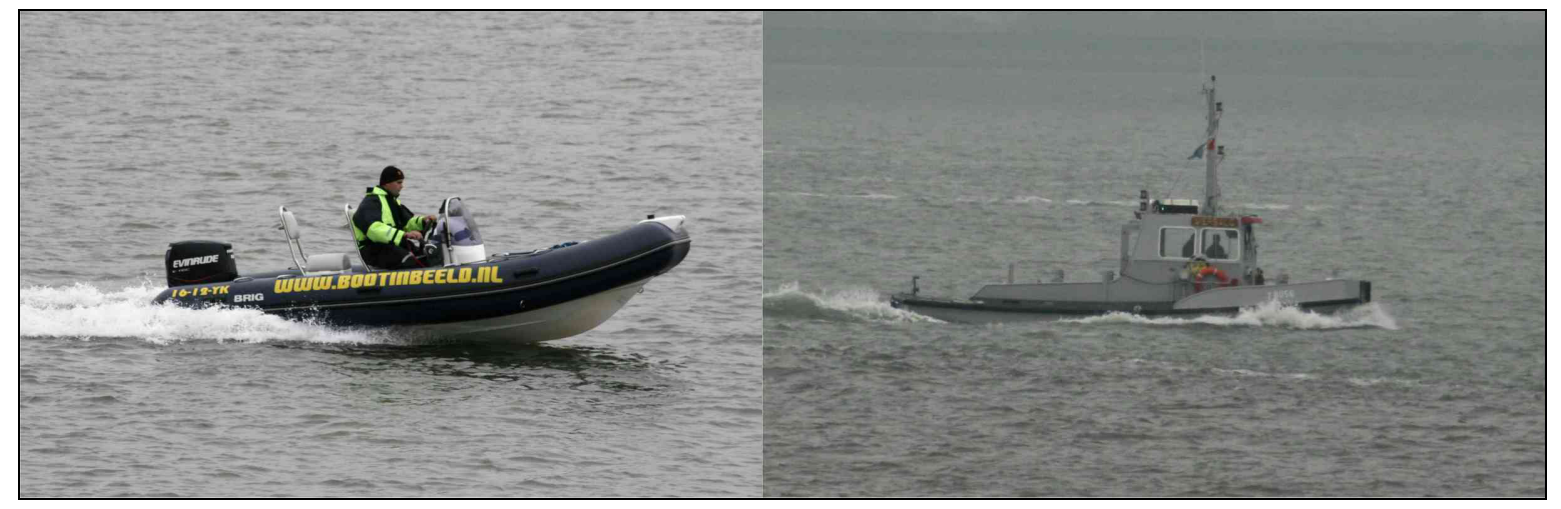

Fig. 6 RHIB (left) and small patrol vessel (right)

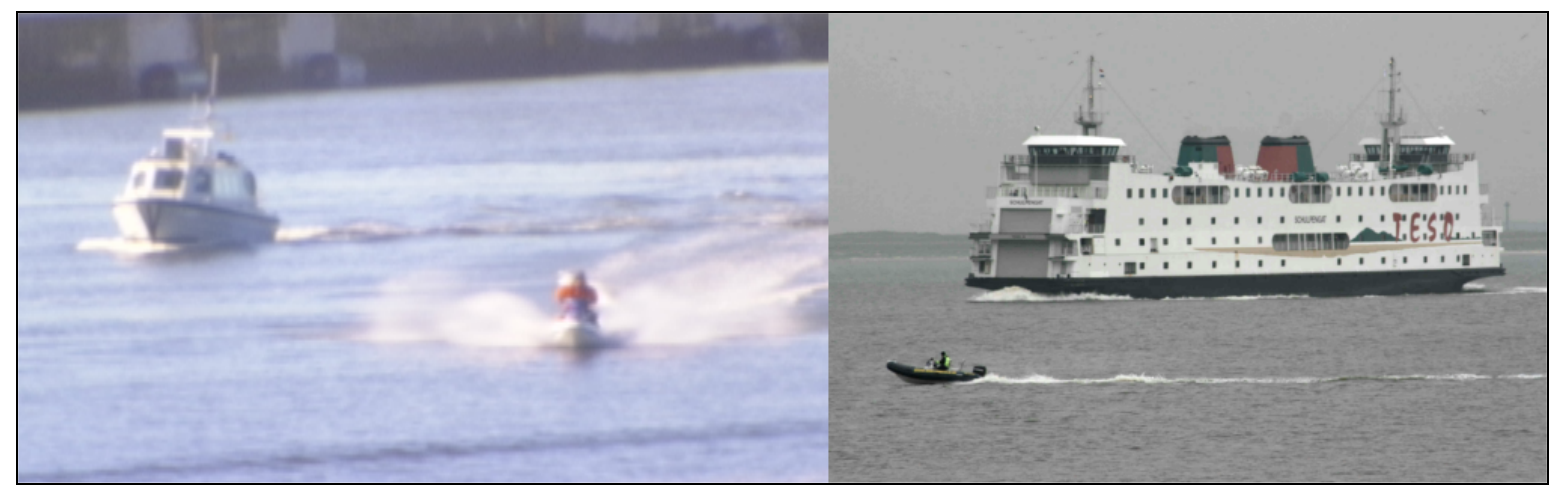

Fig. 7 Patrol boat and jet-ski in harbor (left image); RHIB and ferry between Den Helder and Texel (right image).

\subsection{Cameras}

In order to be able to develop and test algorithmic techniques to detect, track, classify and identify small surface targets in real-time a data collection trial was organized. Target data were collected with a set of electro-optical cameras, radar and GPS. The cameras were mounted on a tripod on the roof of a building at $9 \mathrm{~m}$ above sea level.

The set of cameras consisted of two long-wave infrared (LWIR) cameras with a wide field-of-view (WFOV) and 5 cameras with a narrow field-of-view (NFOV). Details of the cameras are tabulated in Table 1.

Table 1 Details of the cameras.

\begin{tabular}{|l|l|l|l|l|l|l|}
\hline System & Type & Lens & Array & FOV & IFOV [mrad] & Output \\
\hline WFOV LWIR & Vosskühler IRC 320 & $18 \mathrm{~mm}$ & $320 \times 240$ & $35 \times 26^{\circ}$ & $1.9 \times 1.9$ & Digital \\
\hline NFOV MWIR & Radiance HS & $75 \mathrm{~mm}$ & $256 \times 256$ & $6.0 \times 6.0^{\circ}$ & $0.4 \times 0.4^{\circ}$ & Digital \\
\hline NFOV LWIR & AIM QWIP & $250 \mathrm{~mm}$ & $640 \times 512$ & $3.9 \times 3.0^{\circ}$ & $0.11 \times 0.11$ & Digital \\
\hline NFOV VIS (hot) & Marlin F-033B & $75 \mathrm{~mm}$ & $640 \times 480$ & $4.9 \times 3.7^{\circ}$ & $0.13 \times 0.13$ & Fire wire \\
\hline NFOV NIR & Merlin EM-247 & zoom & $320 \times 240$ & $7.9 \times 5.9^{\circ}$ & $0.4 \times 0.4$ & USB \\
\hline NFOV color & Hitachi HV-C20 & zoom & $752 \times 582$ & $3.0 \times 2.2^{\circ}$ & $0.07 \times 0.07$ & Analog \\
\hline
\end{tabular}

Note: The Marlin F-033B camera was fitted with an Andover 775FW82 $(\varnothing=24 \mathrm{~mm})$ HOT filter, cutting off the near infrared wavelengths. The Raptor Photonics Merlin EM-247 EMCCD camera was fitted with an infrared transmitting Black Glass filter (Melles Griot 03FCG509), cutting off the visible wavelengths. 


\section{CLASSIFICATION PROCESSING}

A detection algorithm [9] provides us with bounding boxes in the image encapsulating a ship. For such a region of interest, the image part is extracted. Ships are detected at various sizes and distances. First the image is scaled to a predefined number of pixels to achieve scale invariance. The ship image is subsequently described by computing features.

Ships can be seen under arbitrary viewpoints. Hence, to limit the number of examples that are necessary to obtain a good classification, the description of the ship images needs to be robust to small affine transformations. The approach taken in this paper is to describe the ship image as a set of localized features [13]. Each of the features is localized, which makes it more feasible to achieve some robustness at the level of the smaller image patches. Furthermore, the description by multiple localized features makes the approach more robust when only a part of the image is observed. This is beneficial if some parts of the ship have low contrast due to transmission effects, clutter or partial occlusion. From a technical perspective, the local features have the advantage that no full segmentation (i.e. a very complex task) is required.

For description, various feature types are considered:

- Moments: statistical features that describe implicitly properties such as symmetry and skew [14]. This is informative of the local intensity distribution. Three types of moments are considered in our setup: Global, central moment: one feature computed from the total image (no interest points are used; only included as a reference to standard performance). Local, central moment: the same feature as above, but centered around the interest points. Local, Humoments: the same as above, but with additional invariant properties, such as rotation invariance.

- Gaussian image derivatives: filter-based gradients of the image, indicative of edges and curves [15].

- Gabor texture measurements: Gabor filters perform a local Fourier analysis, hence indicative of local texture [16].

Statistics: for four orthogonal orientations (left, right, up, down) the numerical matrix gradients from the image part are collected. Inspired by SIFT [17] but a simpler feature hence computationally more efficient. For each orientation, corresponding gradients are extracted which are weighed by their gradient magnitude. Hence, only statistics of strong edges are gathered. For instance, if an image part contains a white bar above a dark bar, the magnitudes will be predominantly oriented upwards. A 4-valued feature vector of orientations in a local neighborhood is thus obtained; it can be considered as a simplified SIFT-feature.

An example of the classification results, on similar data as recorded during the trials, is given in Fig. 8, where a water taxi, a jet-ski and a RHIB are correctly classified with the described classification processing.

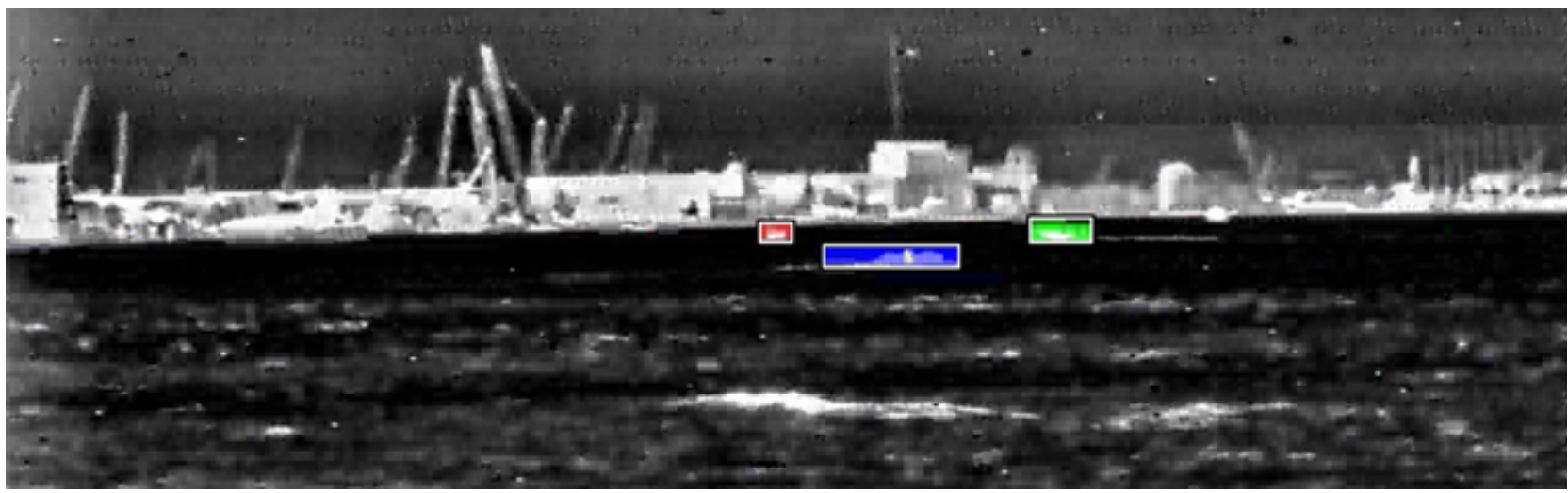

Fig. 8. Frame from a sequence processed by the processing chain described in Section 2.1, including classification based on global descriptors. Color labels indicate classified type. Water taxi (red, left), jet-ski (blue, middle) and RHIB (green, right) are all classified correctly. 


\section{RESULTS}

Existing approaches for enhancing situational awareness are mainly focused on enlarging the observable field-of-view by stitching captured images or different camera views together. In this section we propose two alternative techniques that go beyond creating a single overview image. The first technique is warping image data to absolute coordinates and the second is combining multi-resolution images. Furthermore we show that two color representations of a multiband fused image can enhance situational awareness.

\subsection{Combining wide and narrow field-of-view cameras in panoramic imaging}

Building a $360^{\circ}$ panoramic image requires wide field-of-view cameras. Even if high-resolution image sensors are used, the distance at which targets can be detected or recognized will be limited due to the required large field-of-view. Naval vessels often utilize other camera systems with a narrow field-of-view to observe targets at larger distances. These cameras are mounted on pan-and-tilt units in order to be able move the camera's field-of-view around and track targets. However, it is often difficult for an operator to keep track of the camera's azimuth and elevation based only on visual references.

A better solution would be to add the narrow field-of-view image to the panorama image obtained from the wide fieldof-view cameras. In this case, the panoramic image formed by the wide field-of-view cameras can also be used to direct the narrow field-of-view (zoom) camera to targets. However, due to the wide field-of-view, there is a limit to the resolution in which the target can be observed. This limit can obscure important details required for recognition proposes.

\subsection{Multiband image fusion}

Currently, multi-band and hyper spectral imaging sensors in the thermal infrared are under development. These systems promise significant improvements in military task performance. With these new systems, targets may be distinguished not only on the basis of differences in radiation magnitude, but also on differences in spectral properties. A problem with presenting multi or hyper spectral imagery to a human observer is the (huge) amount of information. The question is how the data should be made available to the human visual system, i.e. which presentation offers the best information transfer. This also depends on the task at hand (e.g. detection, situational awareness, identification) and the prior information available [18].

In this paper we used two color representations to visualize a multiband image of a scene that occurred during the trial. The multiband consisted of four images of a frigate docked in the harbor with a group of people standing on the bow (Fig. 9). The four images were acquired by a near infrared camera (image left), a mid-wave infrared camera (second image left), a long-wave infrared camera (third image left) and a color camera (image right). In order to get the color representations first a principal component transformation was carried out. Next, the first three principal components were assigned with hue, saturation and value signals. The result image is depicted in Fig. 10 (left image). In this image there is a clear distinction between the people on the bow (dark dots on the left), the frigate, building, parking lot and the sky. Also sun reflections such as arriving from the parked cars are clearly visible (dark blobs on the lower right). In the right image of Fig. 10 the hue and saturation were taken from the color image. This color representation appears more natural. Now, the trees and grass are better visible. Both representations can enhance the situation awareness.

At night, when no daytime image is available, a recently developed technique for adding natural daytime colors to nighttime imagery can be used to enhance situational awareness and create a more intuitive image $[19,20]$ 


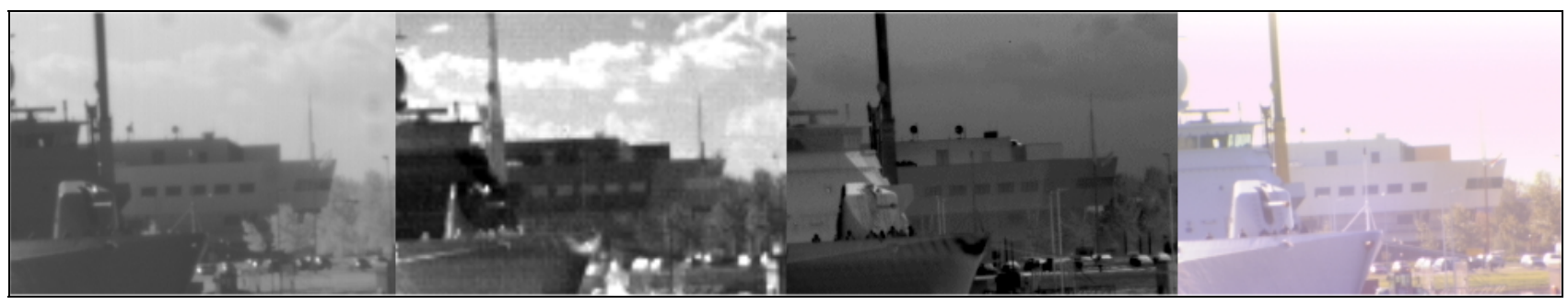

Fig. 9 Images of a frigate docked in the harbor with a group of people standing on the bow. The images are acquired by a near infrared camera (first image left), mid-wave infrared camera (second image left), long-wave infrared camera (third image left) and a color camera (image right).

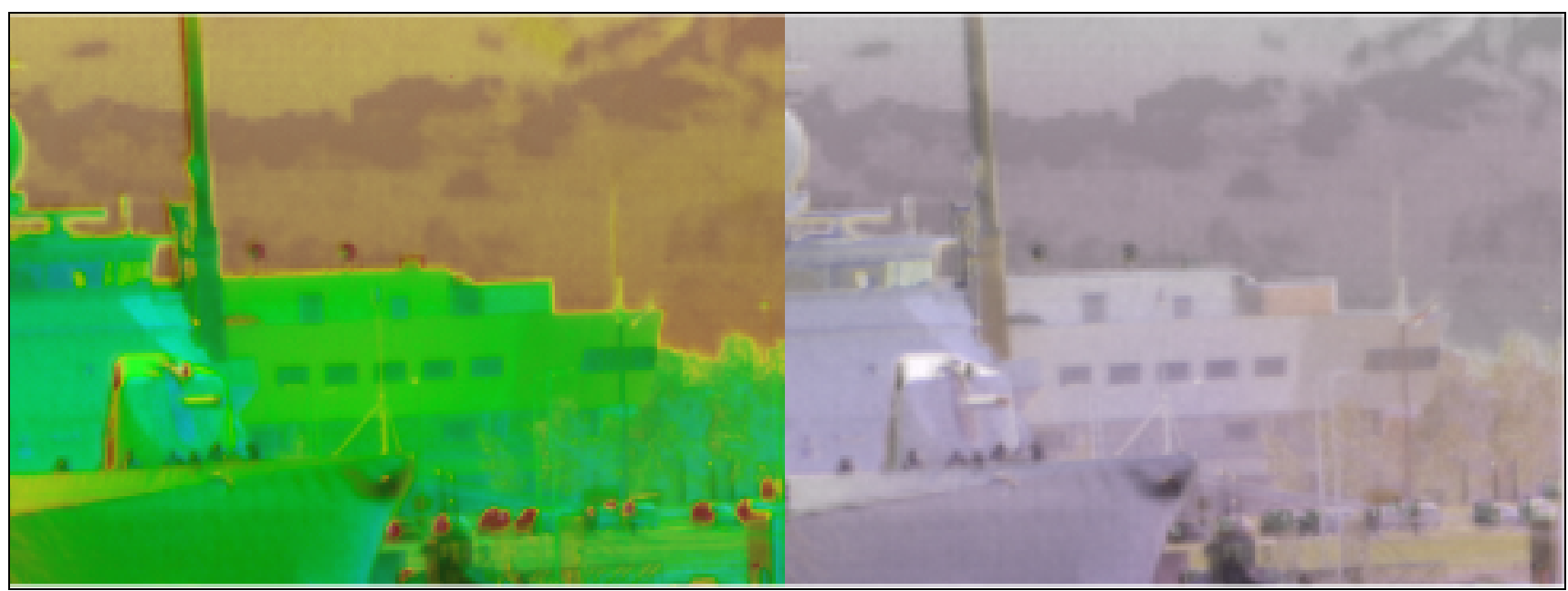

Fig. 10 Color representation of a multiband image using the first three principal components as hue, saturation and value signals (left). The multiband image combines a near infrared, mid-wave infrared, long-wave infrared and color image. In the image right the hue and saturation is obtained from the color image.

\section{CONCLUSIONS}

In this paper a trial was described to collect data with the purpose to demonstrate the feasibility of new techniques and approaches to detect, track, recognize and identify small surface targets in and near a harbor environment. It was demonstrated that a sensor network could be realized consisting of a heterogeneous set of cameras with panoramic observation and zoom capabilities. The data collected using this network was completed by radar, GPS, AIS, synoptic and radiosonde weather data. During the trial data had been collected of small surface targets in and near the New Harbor of Den Helder, in the Netherlands, in October 2008.

In this paper the application of a heterogeneous panoramic sensor and it's application in a harbor environment have been discussed. The combination of such a sensor combined with a high-resolution narrow field-of-view sensor has been demonstrated. Acquiring and fusing detailed imagery for classification whilst keeping an eye on the complete situation around your platform provides a major advantage. Combining the strength of overview and detail simultaneously brings constant situational awareness to future observation systems. A sensor package consisting of a static panoramic camera, combined with a fast narrow field-of-view heterogeneous camera set seems feasible.

In addition to the trial description some examples are given of new approaches to present target image data. These new approaches included combining multi-resolution and multiband images. The first approach combines detailed narrow field-of-view images obtained from a pan-tilt and zoom camera with those from a wide field-of-view panoramic camera system. All image data is merged into a single display, which allows for seamless panning, tilting and zooming between the panoramic view and the detailed narrow field-of-view of the zoom camera. This concept will demonstrate how to simultaneously keep an panoramic overview of the situation with the ability to zoom in on details for classification 
purposes. The second approach combines six multiband images into one fused image with two different color representations. These representations can enhance the situation awareness, by selecting the optimum image data.

Concerning classification, results were presented in this paper on ongoing work on discriminating classes of small vessels recorded with electro-optical systems. A method using global descriptors of segmented objects is implemented in software that combines data-acquisition, detection, clutter reduction, feature extraction and tracking with this classification, resulting in tracks labeled as one of several classes of small vessels. This implementation allows nearrealtime processing of data, and is applied to real infrared recordings of small vessels such as RHIB, jet-ski and water taxi (cabin boat).

Various local descriptors (based on keypoints) have been evaluated for the representations of ships. Evaluation was based on classification accuracy and computational efficiency. If ships are expected to be seen at orientations within 45 degrees of side views, Gaussian features are to be preferred. They are cheap in processing demand and perform well. When more training examples are included in our database, the Gaussian features work well. If less training images are included in the database, the late fusion algorithm, which combines all features in an appropriate weighing scheme, is to be preferred as it is most robust. However, it is a computationally more expensive method. Most time of classifying one image is spent on computing the image features. Gaussian features are computationally cheap and provide the most significant speed-up.

A single measurement does not allow declaring an object to be of a certain class, even with high classification probability for that class, if prevalence of different classes is not taken into account. To make classification useful for an operator, false positives need to be reduced by improving correct classification of the more common classes. Another way is to combine several observations in time, reducing the probability of a classification as being a false positive.

\section{ACKNOWLEDGEMENTS}

This work was financially supported by the Netherlands Ministry of Defense with their research program V602 on Electro-Optical sensor systems. The trial described in this paper was part of a technology project co-funded by the Ministry of Defense and the Ministry of Economic Affairs. Further we acknowledge the hospitality of the Naval Maintenance Establishment of the Royal Netherlands Navy for arranging the trial site and targets. Moreover, we acknowledge the valuable radiosonde measurements carried out by the Meteorological and Oceanographic Centre (METOC) of the Royal Netherlands Navy.

\section{REFERENCES}

[1] Commercial Crime Services (CCS), http://www.icc-ccs.org

[2] "USS Cole (DDG 67), Command History", http://www.cole.navy.mil, October 12, 2000.

[3] "USS Cole bombing", http://en.wikipedia.org/wiki/USS_Cole_bombing.

[4] Theije, P., de, Kester, K., Bergmans, J., "Application of the M6T Tracker to Simulated and Experimental Multistatic Sonar Data”, Fusion 2006, Florence, Italy, July 2006.

[5] Valkenburg-van Haarst, T., Norden, W. van, Bolderheij, F., "Automatic sensor management: challenges and solutions”, in 'Optics and Photonics in Global Homeland Security IV', eds. Halvorson, C.S., Lehrfeld, D., Saito, T.T., SPIE Vol. 6945, Published 17 April 2008.

[6] Schwering P.B.W., Broek S.P. van den, Iersel M. van, "EO System Concepts in the Littoral", in 'Infrared Technology and Applications XXXIII', eds. Andresen, B.F., SPIE Vol. 6542-100, 654230, Orlando Florida (USA), April 9-13, 2007.

[7] Ditzel, M., Lageweg, C., Janssen, J., Theil, A., "Multi-target Data Aggregation and Tracking in Wireless Sensor Networks", Journal of networks, 2008.

[8] Broek, S. P. van den, Bouma, H., Degache, M. A. C., Burghouts, G., "Discrimination of classes of ships for aided recognition in a coastal environment", SPIE Paper No. 7335-32, in press, 2009. 
[9] Bouma, H., Lange, D.J.J. de, Broek, S.P. van den, Kemp, R.A.W., Schwering, P.B.W., "Automatic detection of small surface targets with electro-optical sensors in a harbor environment," Proc. SPIE 7114, 711402 (2008)

[10] Broek, S.P. van den, Bouma, H., Degache, M.A.C., "Discriminating small extended targets at sea from clutter and other classes of boats in infrared and visual light imagery," Proc. SPIE 6969, 69690B (2008)

[11] Hupkens, T.M., "Invariant Legendre and Zernike Moments," Recent research developments in pattern recognition 3, 35-52 (2002)

[12] Duin, R.P.W., Juszczak, P., Paclik, P., Pekalska, E., de Ridder, D., Tax, D.M.J., "PRTools4, A Matlab Toolbox for Pattern Recognition," http://prtools.org, Delft University of Technology (2004)

[13] Mikolajczyk, K. and Schmid, C., "A performance evaluation of local descriptors," IEEE Trans. Pattern Analysis and Machine Intell. 27(10), 1615-1630 (2005)

[14] Hu, M.K., "Visual pattern recognition by moment invariants," IRE Transactions on Information Theory 8, 179187 (1962)

[15] Koenderink, J.J., "The structure of images," Biological Cybernetics 50, 363-370 (1984)

[16] Bovik, A.C., Clark, M., Geisler, W.S., "Multichannel texture analysis using localized spatial filters," IEEE Transactions on Pattern Analysis and Machine Intelligence 12, 55-73 (1990)

[17] Lowe, D.G., "Distinctive image features from scale-invariant keypoints," International Journal of Computer Vision 60(2), 91-110 (2004)

[18] Hogervorst, M.A., Bijl, P., Toet, A., "Visualization of hyperspectral imagery", in 'Algorithms and Technologies for Multispectral, Hyperspectral, and Ultraspectral Imagery XIII', eds. Shen, S. S. \& Lewis, P. E., SPIE-6565-16, 2007.

[19] Hogervorst, M.A., Toet, A., "Method for applying daytime colors to nighttimeimagery in realtime", in 'Multisensor, Multisource Information Fusion: Architectures, Algorithms, and Applications 2008', eds. Dasarathy, B.V., SPIE-6974, 2008.

[20] Hogervorst, M.A., Toet, A., "Fast natural color mapping for night-time imagery", Information Fusion, (in press) 2008. 